\title{
Organizing a Webinar during a Viral Pandemic
}

\author{
Nupur K Nerurkar ${ }^{1}$, Gauri M Kapre ${ }^{2}$, Frederik G Dikkers ${ }^{3}$
}

\begin{abstract}
Aims and objectives: The coronavirus disease-2019 (COVID-19) pandemic has changed lives and caused great loss and hardship to many people around the world. Not wanting to break the chain of our annual Phonosurgery Workshop, the decision was taken to plunge into the virtual world. This article aims to share the challenges faced by the organizing committee and the advantages (some unexpected) and limitations of this academic venture, which may encourage and possibly guide our colleagues in hosting their own meetings.

Materials and methods: Several major decisions were taken: (1) create a 1-day event instead of the usual 3 days; (2) include a maximum number of invited faculty from abroad; (3) select very specific areas of interest; (4) employ a professional online communications group; (5) broadcast prerecorded presentations; (6) include a live $\mathrm{Q}$ and $\mathrm{A}$ session immediately following each lecture; (7) incorporate audience polling, e-posters, e-crossword, e-stalls, and live chat communication.

Results: The average number of delegates increased from 197 to 1071 delegates. The number of international delegates was much higher. Since no "physical" space for the delegates was needed, the number of registrations did not need to be restricted.

Conclusion: The decisions described above had a positive impact on the number of registrations, without hampering the quality of the course. The experience of hosting this webinar has encouraged the organizing committee to consider a hybrid meeting in the year 2021 with both physical and virtual attendance.

Keywords: Course organization, COVID-19 infection, Laryngology, Organizing committee, Webinar.

International Journal of Phonosurgery \& Laryngology (2021): 10.5005/jp-journals-10023-1193
\end{abstract}

\section{INTRODUCTION}

Coronavirus disease-2019 (COVID-19) pandemic has changed lives and caused loss and hardships to people around the world. However, human beings are capable of adapting and innovating. Though the medical fraternity has had to tackle treating patients during this pandemic as well as taking care of their families, the academic initiative has not ebbed but taken on new formats. The authors would like to share their experience with arranging the 14th Workshop on Phonosurgery as a Webinar during the pandemic. Not wanting to break the chain of the annual phonosurgery workshops, the decision was taken to plunge into the virtual world. Around the same time some other ENT conferences globally also decided to go virtual [e.g., 13th Congress of the European Laryngological Society (Germany), Combined Otolaryngology Spring Meeting (USA)], while others decided to skip a year or two (6th Congress of European ORL-HNS (Italy)).

Our annual workshops are held as 3-day meetings encompassing lectures on the first day, live surgery on the second, and hands-on workshops (fresh frozen cadaver dissection, stroboscopy, and dysphagia workshop) on the third. Typically, a few international faculty and 10-15 national faculty are invited and an average of 197 delegates from India and neighboring countries attend. An award poster session is held every year with the posters displayed as hard copies.

This article aims to share the challenges faced by the organizing committee and the advantages (some unexpected) and limitations of this academic venture. This may encourage and possibly guide colleagues in hosting their own meetings.

\section{Materials and Methods}

Originally, our meeting was scheduled to take place from September 11 to September 13, 2020. Due to the COVID pandemic, in April 2020
${ }^{1}$ Head-Bombay Hospital Voice and Swallowing Center, Bombay Hospital and Medical Research Centre, Mumbai, Maharashtra, India

${ }^{2}$ Department of ENT, Neeti Clinics, Nagpur, Maharashtra, India

${ }^{3}$ Department of Otorhinolaryngology, Amsterdam UMC, University of Amsterdam, Amsterdam, The Netherlands

Corresponding Author: Nupur K Nerurkar, Head-Bombay Hospital Voice and Swallowing Center, Bombay Hospital and Medical Research Centre, Mumbai, Maharashtra, India, Phone: +91 9821034085, e-mail: nupurkapoor@yahoo.com

How to cite this article: Nerurkar NK, Kapre GM, Dikkers FG. Organizing a Webinar during a Viral Pandemic. Int J Phonosurg Laryngol 2021;11(1):1-4.

Source of support: Nil

Conflict of interest: None

we started considering "to go digital", and by May, several major decisions were taken (Table 1).

The first decision taken by the organizing committee was to make the e-webinar into a crisp 1-day event to be conducted on a Sunday. In our opinion, a virtual meeting spread over 3 days would be hard to hold the attention of the delegates and make timings difficult to co-ordinate globally.

Our next decision was to include a maximum number of international faculty to create an inclusive meeting at a global level. A total of nine international [from New York, Seattle, Birmingham, San Francisco, Los Angeles (USA), Hamburg (Germany), Amsterdam (The Netherlands), and (Luxembourg)] and four national faculty were invited. Our next task was to make sure that the time zones were possible for our faculty all across the world. We started our meeting at 8 am IST in India with the speakers from the east coast of the USA (where it was 10:30 pm EST of the previous day) coming 
Table 1: Summary of general decisions taken to organize a successful international meeting

\begin{tabular}{ll}
\hline 1 & Create a 1-day event \\
2 & Include a large international faculty \\
3 & Select specific areas of interest \\
4 & Employ a professional online communications group \\
5 & Broadcast prerecorded presentations \\
6 & Include a live interactive session following each lecture \\
7 & Incorporate audience polling, online crossword, and e- \\
\hline
\end{tabular}

on first, followed by the west coast an hour later (9:30 pm PST the previous day), moving into Europe (7:30 am GMT same day) next, and finally India where there are no time zones.

To gain maximum exposure and attendance, website creation (www.phonosurgeryworkshops.com) was initiated in March 2020 detailing faculty, presentations, and the scientific program and this website remain active.

To help us seamlessly transcend to the virtual platform, we decided along with our event management team, to employ a professional online communications group based out of Dubai but with offices in Pune, India. Our entire event was then transmitted from their studio. On the day of the event, the entire organizing team and all faculty were at their own residence or office and only 2 members of the event management team joined the broadcasting team at Pune. We decided to charge a very nominal registration fee (INR 500 or $\$ 6.74$ ) to not just cover the operational costs at our end, but also to drive the attendees in all earnest to log in. The payment was possible online through our website making the process simple.

To avoid loss of time and maintain a smooth flow of sessions, we requested all faculty to prerecord their talks, preferably in a format that showed the speaker's face alongside the PowerPoint presentation. The advantage of having everything prerecorded was that the timings were exactly accounted for. By relaying everything from a one-point source, there was no waste of time in trying to "share screens" by presenters, no problems with videos not playing or audio not audible via personal computers dependent on varying Wi-Fi networks and varying software upgradation. The feel for the delegate was just slightly different compared with a live lecture as the speaker's face was appreciable as a picture-in-picture (Fig. 1). To guide faculty regarding prerecording options, detailed instructions and guidelines were emailed in a simple illustrative Power Point presentation for prerecording techniques for Zoom ${ }^{\oplus}$, Mac iOS, and Windows format. Our technical support team was readily available for any faculty facing difficulty and did actually carry out the recording over Zoom ${ }^{\circledast}$ for one of our European faculty.

To keep the meeting as "live" as possible, the $\mathrm{Q}$ and $\mathrm{A}$ session was in real-time and the speakers could log in to the studio link (exclusive for faculty) and come on "live" after their prerecorded talks were aired. The broadcast studio could be accessed only by the faculty and once they logged in, the studio personnel would keep them in a "waiting room" where they could check their audio and video feed clarity and only then allow them to "enter" the studio and come on live on screen. This ensured that there was no incident of someone's audio/video feed being accidentally turned on or off without the studio's control. Participants could send in their questions using the chat function on the webinar link. A designated moderator could pass on these to the presenters. Presenters could also read the questions, apart from just listening to them, which

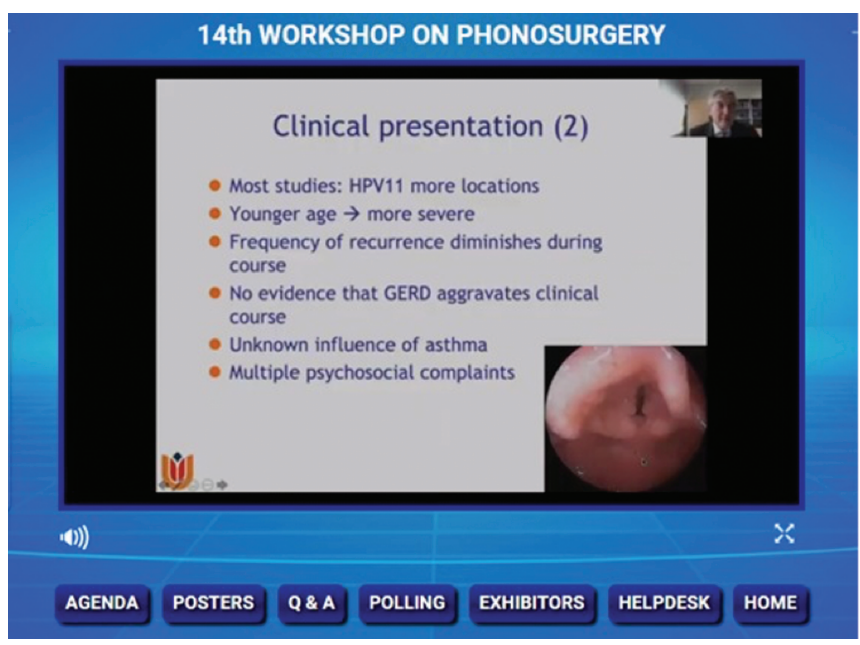

Fig. 1: Delegate view showing the speaker seen as "picture in picture" in the presentation

made it easy to understand and avoid the occasional problem of not understanding different accents between American, Dutch, French, German, and Indian English. The panel was conducted live with the European and Indian faculty after the meeting, 3:30 pm IST in India, 12 noon GMT in Europe.

Teaming up with a professional communication platform allowed us to add certain unique features to our webinar, which enhanced the attendees' experience. We could conduct live polling to questions at the end of each talk which made the whole session interactive and allowed the speakers to gauge how the audience had received their talks. Another feature we added was a live "laryngology" crossword, which was an online game for the participants to play during the mid-day break. The results of the crossword were updated in real-time and could be viewed by all with the first five correct entries receiving prizes.

The posters were invited as e-posters with 3-minute audio files of the presenters explaining them. A selection panel selected the 12 final posters out of a total of 38 posters received. All 38 e-posters were displayed on our website and on the final day the selected 12 e-posters were displayed as short films with audio running.

After the webinar, a feedback questionnaire was sent out to all participants.

\section{Results}

The average number of delegates increased from 197 over the past years, to 1,071 delegates this year. The International delegate participation increased from $1.5 \%$ in the previous year to $7.0 \%$ (Fig. 2). The average International faculty in the previous years was $5 \%$ and this year the composition was $69 \%$ of international faculty. The poster submissions were 38, compared with an average of 16 posters in the previous years. The registration cost for the 1-day meeting was INR 500 or $\$ 6.74$ compared with an average daily cost of INR $3000 / \$ 40.4$ in the previous years. There were no live surgical demonstrations, unlike previous years.

The feedback received was extremely positive with the one critical comment being a very busy scientific program.

\section{Discussion}

In our opinion, an online event holds several advantages over a live event. In our earlier workshops, the average number of delegates 


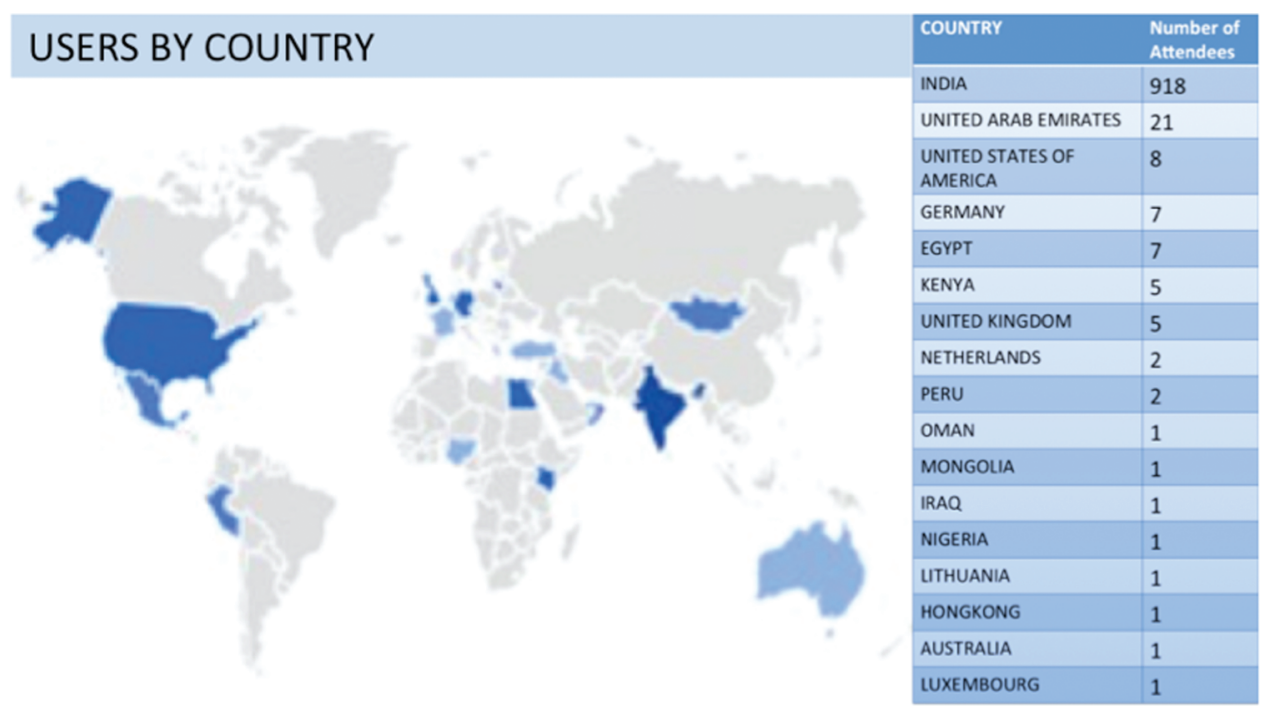

Fig. 2: Distribution of delegates logged in during the 14th Workshop on Phonosurgery around the world. From light blue to dark blue indicating the density of delegates, dark blue being the most number of delegates logged in, white being areas with no delegates logged in. The adjoining table shows the details of number of delegates per country

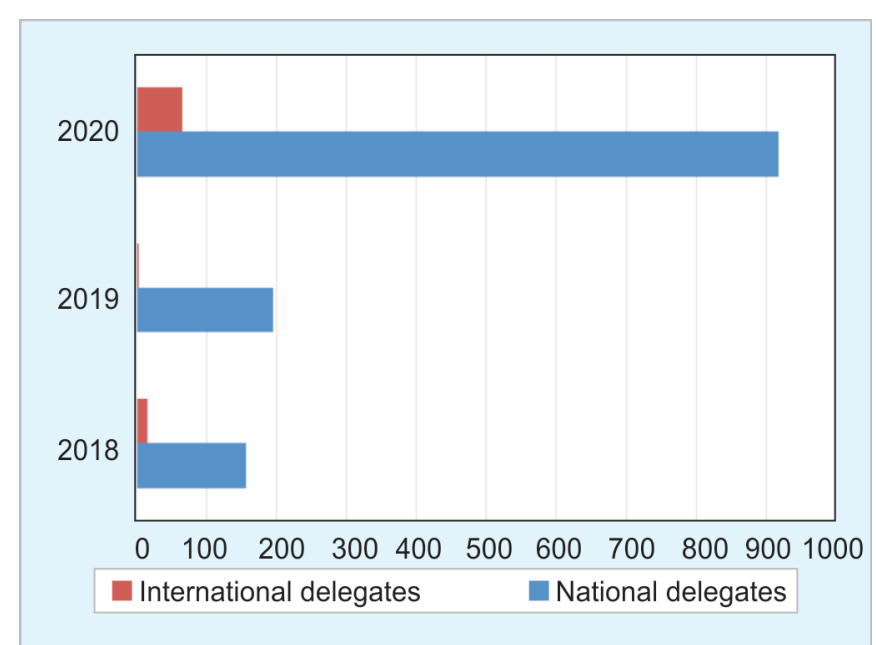

Fig. 3: Annual distribution of number of International and National delegates to the "Workshops on Phonosurgery", from the past 3 years

was 197, whereas this year, we had 1,071 delegates registered (983 devices were actually logged in on the day) (Fig. 3). This number was significantly higher than our previous year's attendance. Furthermore, the number of international delegates was much higher which is due to the advantage of not having to travel. A third advantage an online event offered was that since we did not need any "physical" space for delegates, we did not have to restrict the number of registrations, which can be an issue sometimes due to space constraints at the conference venue. As some of our delegates pointed out, those who were working at home or in the hospital, could still log in and listen to the lectures without having to take time off. Most of the delegates logged in using their phone (69.8\%), while others on their desktop or tablet (27.1 and 3.1\% respectively); though the experience on the computer screen was better as given in the feedback by delegates who had used both modalities at different times.

The organizing committee had a set of backup plans in place for every step. Having an experienced IT and broadcast team in place, helped the committee overcome technical difficulties.
Multiple trial runs were conducted with the broadcast studio, speakers, chairpersons, and event management team to iron out every foreseeable glitch.

Another challenge we encountered was holding onto the delegates' attention for an entire day. With limited interpersonal interaction, focusing on an online event for an entire day does become difficult. With the addition of polling questions, crossword puzzles, and live Q and A sessions, we tried to keep it as "alive" and interesting as possible.

The one irreplaceable advantage a live event boasts is, the possibility of viewing live surgery being performed. This has become a norm in almost all national workshops in India, including our own annual workshop. Attending delegates, particularly younger surgeons, benefit by seeing an operation being performed live, especially where they have the facility to interrupt the surgeon and ask questions. ${ }^{1}$ However, the flip side to this coin is surgeon-related stress in a live transmission setting and patient permissions. ${ }^{2}$ Additionally, surgeons are probably most at ease operating the comfort of their own operation theater (OT) rather than the conference venue OT, which they are unfamiliar with, and using equipment they are possibly unaccustomed to. In the first European Laryngological Live Surgery Broadcast in 2015, surgeons performed complex surgical procedures at the surgeon's home institute, to eliminate the issue surrounding an unfamiliar environment. ${ }^{1}$ It might be hypothesized that surgery performed at a foreign setup might not be able to reflect the surgeon's true technique and outcomes. The inevitable delay that live surgery entails, due to several unpredictable factors being involved, could also be avoided here. Perhaps in the future years, we may consider presenters showing prerecorded "live" surgeries followed by a discussion with the audience or may even consider having live transmission of a surgery being performed in the faculty's own OT being relayed to our audience, similar to the annual live surgery broadcast of the European Laryngological Society (http://els. livesurgery.net).

The authors understand that this narration is an anecdotal experience. But the unique situation the world finds itself in, with a global pandemic marring scheduled annual conferences; warrants solutions. The authors' intention here is to share their 
solutions with the hope that it may benefit others planning similar events.

\section{Conclusion}

The experience of hosting this webinar has encouraged the organizing committee to consider a hybrid meeting in the year 2021 with both physical and virtual attendance. Faculty participants will be considered in both a physical and virtual manner to allow for more international representation with the possibility of relaying live surgery followed by discussion. The authors expect that readers can avoid negative, and implement positive experiences of our organizing committee in their future webinars.

\section{Meeting Information}

Accepted for Poster presentation at the ABEA Virtual meeting of COSM 2021.

\section{References}

1. Dikkers FG, Bernal-Sprekelsen M, Klussmann JP, et al. ELS live surgery: a developing story. Eur Arch Otorhinolaryngol 2019;276(3):897-899. DOI: 10.1007/s00405-018-05278-4.

2. Dikkers FG, Klussmann JP, Bernal-Sprekelsen M, et al. Live surgery broadcast: who is benefiting? Eur Arch Otorhinolaryngol 2016;273(6):1331-1333. DOI: 10.1007/s00405-016-3977-6. 